\title{
PREVALENCIA DE ALTERACIONES EN LA AGUDEZA VISUAL, DISCROMATOPSIA Y SIGNOS Y SÍNTOMAS ASOCIADOS EN UNA POBLACIÓN ESTUDIANTIL EN LA COMUNIDAD DE EL CARRETÓN, PERAVIA, REPÚBLICA DOMINICANA, DURANTE EL PERÍODO OCTUBRE-DICIEMBRE DEL AÑO 2019
}

\section{Prevalence of alterations in visual acuity, dyschromatopsia and associated signs and symptoms in a student population in the community of El Carretón, Peravia, Dominican Republic, during the period October-December of the year 2019}

\section{Rosa Katherine Rivera Grullóna, Paola Hernándezº , Daniel Bisonóc, Marie Loubeau ${ }^{\mathrm{d}}$ y Amanda García. ${ }^{\mathrm{e}}$}

Recibido: 6 de noviembre, 2019 • Aprobado: 7 de marzo, 2020

Cómo citar: Rivera Grullón RK, Hernández P, Bisonó D, Loubeau M, García A. Prevalencia de alteraciones en la agudeza visual, discromatopsia y signos y síntomas asociados en una población estudiantil en la comunidad de El Carretón, Peravia, República Dominicana, durante el período octubre-diciembre del año 2019. cysa [Internet]. 18 de septiembre de 2020 [citado 18 de septiembre de 2020];4(3):95-104. Disponible en: https://revistas.intec.edu.do/index.php/cisa/article/view/1916

\section{Resumen}

Introducción: la baja agudeza visual es un problema educativo de interés mundial, cuya progresión es evitable con métodos de detección temprana y cooperación activa por parte de los padres y de la institución educativa. El presente estudio tiene como fin definir la cantidad de estudiantes de un centro educativo de El Carretón, Peravia, República Dominicana, que poseen algún tipo de condición que amerite atención oftalmológica especializada, y recalcar la falta de importancia que se le da a la salud oftalmológica en dicha localidad.

Metodología: estudio prospectivo, descriptivo, transversal y observacional en el que se analizó la prevalencia de trastornos de la agudeza visual, discromatopsia y signos y síntomas asociados en una población estudiantil.

Resultados: se puede apreciar que la agudeza visual en el ojo izquierdo fue normal en el $47 \%$ de los casos, siendo

\footnotetext{
a Instituto Tecnológico de Santo Domingo (INTEC). Santo Domingo, República Dominicana. Correo-e: kriverg@hotmail.com

b Instituto Tecnológico de Santo Domingo (INTEC). Santo Domingo, República Dominicana. Correo-e: masiel_hernandez@hotmail.com

${ }^{\mathrm{c}}$ Instituto Tecnológico de Santo Domingo (INTEC). Santo Domingo, República Dominicana. Correo-e: daniel.bisono@hotmail.com
}

\begin{abstract}
Introduction: Low visual acuity is an educational problem of global interest, the progression of which is avoidable with early detection methods and active cooperation by parents and the educational institutions. The purpose of this study is to define the number of students of an educational center in El Carretón, Peravia, Dominican Republic, who have some type of condition that merits specialized ophthalmological care and emphasize the lack of importance given to ophthalmological health in that location.
\end{abstract}

Methodology: Prospective, descriptive, cross-sectional and observational study in which the prevalence of visual acuity disorders, dyschromatopsia and associated signs and symptoms in a student population was analyzed.

Results: It can be seen that visual acuity in the left eye was normal in $47 \%$ of the cases being higher than in the right eye which was $43 \%$ of all the cases; The 8 -year-old

\footnotetext{
'Instituto Tecnológico de Santo Domingo (INTEC). Santo Domingo, República Dominicana. Correo-e: amandaangeles18@gmail.com eInstituto Tecnológico de Santo Domingo (INTEC). Santo Domingo, República Dominicana. Correo-e: bereniceloubeau@yahoo.com
} 
mayor que en el ojo derecho (43\%). Los estudiantes de ocho (8) años de edad tuvieron predominancia en las alteraciones de la agudeza visual en ojo derecho, así como los estudiantes de doce (12) años de edad tuvieron predominancia en las alteraciones en el ojo izquierdo. El $77 \%$ del porcentaje que presentó alteración en la agudeza visual tuvo como síntoma más común la cefalea, y un $10 \%$ no presentó signos y síntomas asociados. Se observó que con el agujero estenopeico hubo mayor mejoría de la visión en el ojo derecho, con un $7 \%$ en comparación con el ojo izquierdo, que fue de un $5 \%$.

Conclusiones: los estudiantes con alteración de la AV presentaron en mayor frecuencia una discapacidad visual leve, tanto en el ojo derecho como en el ojo izquierdo. Los síntomas más asociados a las alteraciones de la vista fueron la cefalea y la visión borrosa. De los signos evaluados, no se evidenció ninguno en la población examinada. Ningún estudiante presentó discromatopsia.

Recomendaciones: realizar campañas de salud pública que enfaticen la importancia de cuidado de los ojos. Involucrar al sistema educativo y a todas las instituciones relacionadas, como aliados en la creación de conciencia sobre la importancia de la identificación de afecciones oculares y el acceso a servicios de atención ocular para estudiantes.

Palabras clave: oftalmología; pediatría; agudeza visual; discromatopsia; comunidad.

\section{Introducción}

La visión es responsable de la mayor parte de la información sensorial que se percibe del medio externo. Desempeńa un papel predominante en los primeros años de vida, al permitir la interacción social, el aprendizaje y la comunicación, de modo que los problemas visuales pueden ocasionar graves perjuicios en el aprendizaje y la socialización. Además, se le ha atribuido como una de las principales causas del fracaso escolar ${ }^{1}$.

La mayoría de los escolares de la comunidad El Carretón, Peravia, no han tenido ningún tipo de acceso a servicios de salud oftalmológica, por lo cual muchos pueden poseer alteraciones de la agudeza students had a predominance in alterations of visual acuity in the right eye as well as the 12-year-old students had a predominance in alterations in the left eye. $77 \%$ of the percentage that presented an alteration in visual acuity had headache as the most common symptom and $10 \%$ had no associated signs and symptoms. It was observed that with the pinhole occluder there was greater improvement of vision in the right eye with $7 \%$ compared to the left eye that was $5 \%$.

Conclusions: Students with VA impairment presented more often a mild visual impairment in both the right eye and the left eye. The symptoms most associated with vision disorders were headache and blurred vision. Of the signs evaluated, none were evident in the population examined. No student presented dyschromatopsia.

Recommendations: Conduct public health campaigns that emphasize the importance of eye care. Involve educational and all institutions related to it, as partners in raising awareness about the importance of identifying eye conditions and access to eye care services among students.

Keywords: ophthalmology, pediatrics, visual acuity, dyschromatopsia, community.

visual sin diagnosticar, que no tratadas representan un riesgo elevado de consecuencias irreversibles para su visión ${ }^{1}$. Según la OMS, al nivel mundial existen aproximadamente 2.2 billones de personas con alteraciones visuales, donde 312 millones de estos corresponden a infantes y adolescentes. ${ }^{2}$

Así como la evaluación de signos vitales es parte de todo examen físico, el examen oftalmológico debe incluir la valoración de la función visual, sin importar si esta no se menciona como parte de la molestia principal ${ }^{3}$. El examen oftalmológico es de suma importancia en la atención de pacientes con síntomas oculares, quienes requieren una evaluación completa y una descripción clínica adecuada ${ }^{4}$. 
Es pertinente realizar una anamnesis integral del paciente, indagando en aquellas enfermedades que padece o ha padecido el paciente, en búsqueda de historial de traumas oculares, antecedentes quirúrgicos e infecciosos, los cuales pueden ser de importancia para determinar el estado de salud oftalmológica actual de dicho paciente ${ }^{4}$.

Muchas enfermedades oculares presentan cierto grado de predisposición familiar, por ejemplo, los defectos de refracción, como la miopía, cuyas primeras manifestaciones se pueden ver reflejadas desde temprana edad y se deben de tomar en cuenta por el médico general ${ }^{4}$. La correcta exploración del ojo es la principal herramienta de diagnóstico en estas patologías.

Para que los estudiantes puedan adquirir el conocimiento de forma adecuada, deben tener una buena salud física y mental, esto hace necesario una correcta evaluación del escolar en lo que a su salud física compete, la agudeza visual no es excepción en este aspecto. La baja agudeza visual es un problema educativo de interés mundial, puede provocar un retraso en el aprendizaje del estudiante, haciéndolos más susceptibles a problemas con sus calificaciones.

Los defectos visuales no son solo asunto de la adultez y de la vejez, sino también compete a aquellos de cortas edades 5 . La progresión de los defectos visuales es evitable con métodos de detección temprana y cooperación activa por parte de los padres y de la institución educativa, que debe velar por el bienestar, rendimiento y desempeño de sus estudiantes.

El presente estudio tiene como finalidad definir la cantidad de estudiantes de un centro educativo de El Carretón, Peravia, República Dominicana, que poseen algún tipo de condición que amerite atención oftalmológica especializada y recalcar la falta de importancia que se le da a la salud oftalmológica en dicha localidad.

\section{Material y métodos}

La presente investigación fue de carácter prospectivo, debido a que la información se obtuvo de fuentes primarias, recolectada de forma directa por los investigadores a partir de los sujetos a investigar. Además, fue transversal ya que las variables fueron medidas en una sola ocasión y no se les dio seguimiento a las mismas; también fue observacional debido a que los investigadores se limitaron a medir las variables sin ejercer control sobre el factor de estudio. Por último, esta investigación fue de tipo descriptivo debido a que evalúa la frecuencia y tendencias de la población con respecto a una enfermedad y a la vez describe un problema de salud en función a las características de persona, lugar y tiempo de aparición.

La población seleccionada fue de 487 estudiantes de una escuela de El Carretón, Peravia, República Dominicana. La muestra obtenida fue de 119 estudiantes, utilizando la siguiente fórmula para variable cualitativa con población conocida:

$$
n=\frac{N z_{\alpha}^{2} p q}{d^{2}(N-1)+z_{\alpha}^{2} p q}
$$

donde $\mathrm{z}=1.96$ (intervalo de confianza de $95 \%$ ), una prevalencia $(\mathrm{p})$ de $\mathrm{p}=27 \%$ y un nivel de precisión o error que se pretende cometer $(\mathrm{d}) \mathrm{de} \mathrm{d}=5$ $\%$. Se estratificó la muestra en función a los grados a que pertenecían los estudiantes de dicha escuela; donde luego se realizó una proporción para indicar cuántos estudiantes iban a ser seleccionados por grado.

Los datos fueron recolectados a través de la aplicación de un cuestionario virtual (véase anexo 1), el cual fue elaborado por los autores de la presente investigación y fue aplicado a cada uno de los estudiantes por parte de los mismos investigadores, a la vez, se realizó un consentimiento informado 
y fue dirigido a cada padre de dichos estudiantes (véase anexo \#2) donde se explicó la total confidencialidad de la información. También se aplicó una tabla de Snellen (para alfabetizados) y una tabla de optotipos pediátricos (para no alfabetizados) con la finalidad de evaluar la agudeza visual y a la vez se aplicó el test de Ishihara de 38 láminas para detectar si había presencia de discromatopsia.

El programa utilizado para la tabulación y análisis estadístico de los datos recolectados fue Microsoft Excel versión Office 2016.

\section{Resultados}

Gráfica 1. Agudeza visual de ojo derecho $(\mathrm{n}=119)$

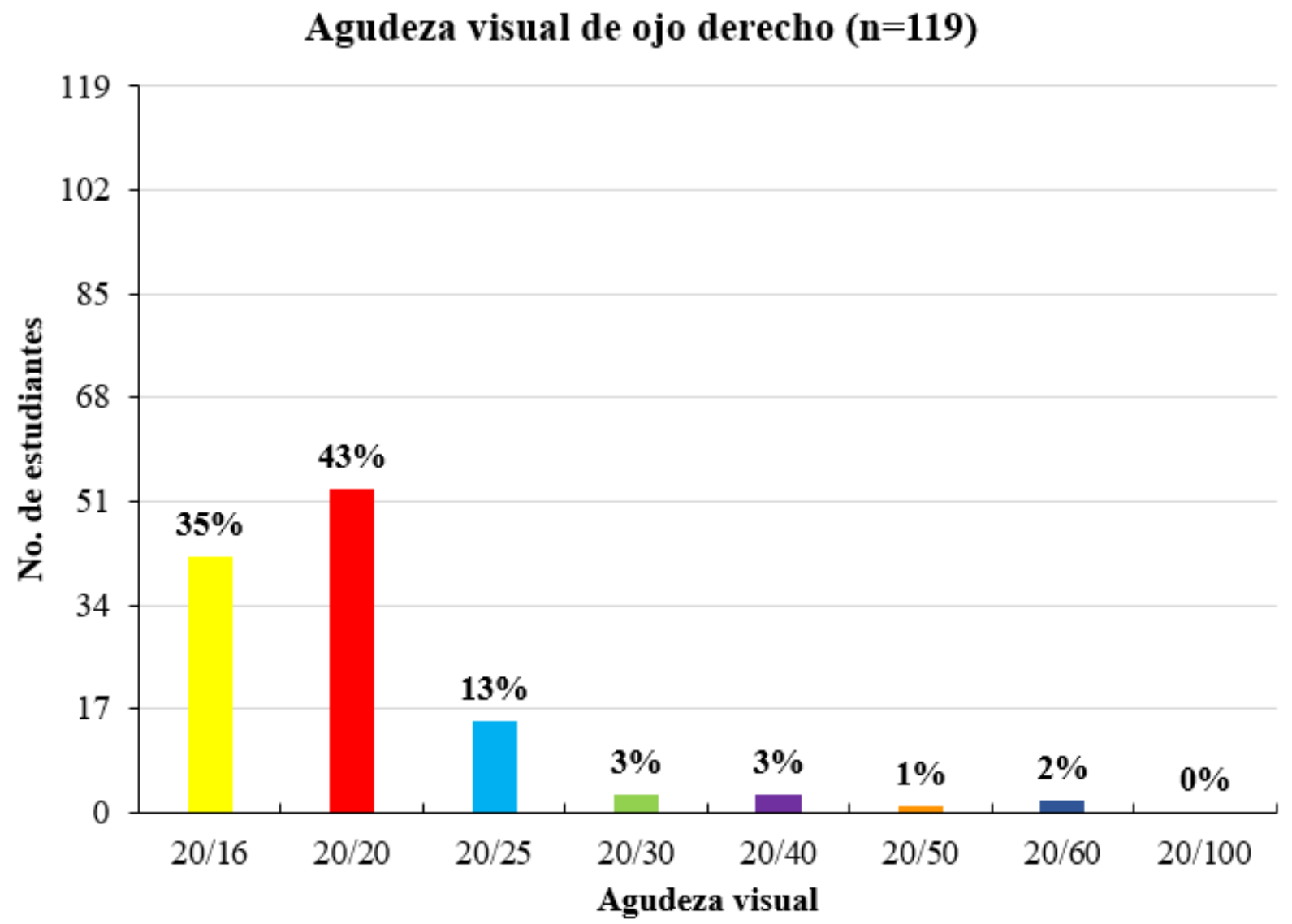

Fuente: Bisonó, García, Hernández y Loubeau. Trabajo de grado, 2019.

En esta gráfica, se pueden apreciar los resultados de los valores de la agudeza visual del ojo derecho de los estudiantes de la escuela de El Carretón. De los 119 estudiantes evaluados, $35 \%$, correspondiente a 42 casos, obtuvo una visión de 20/16; 43 $\%$, equivalente a 53 casos, presentó una visión de 20/20; $13 \%$, correspondiente a 15 estudiantes, obtuvo una visión de 20/25; además, 3 estudiantes, representado por el $3 \%$ de la muestra, poseían una visión de 20/30 y otros 3 obtuvieron una visión de 20/40, también $1 \%$, equivalente a 1 estudiante, obtuvo una visión de $20 / 50$; y $2 \%$, correspondiente a 2 estudiantes, obtuvo una visión de 20/60. Por último, ninguno de los estudiantes presentó una visión de 20/100. 
Gráfica 2. Agudeza visual de ojo izquierdo $(\mathrm{n}=119)$

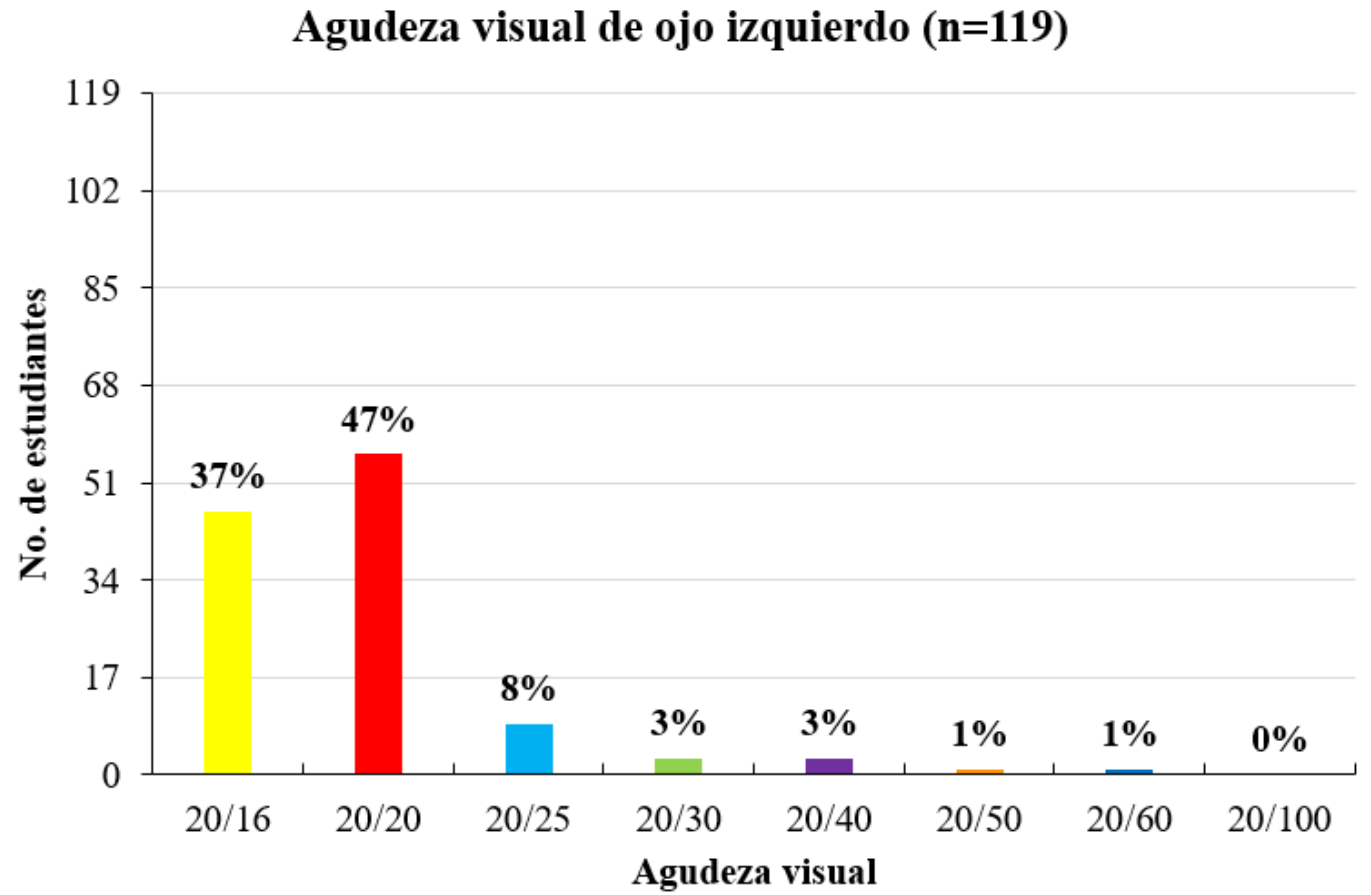

Fuente: Bisonó et al., 2019.

Esta gráfica muestra los resultados de los valores de la agudeza visual del ojo izquierdo de los estudiantes de la escuela de El Carretón. De los 119 estudiantes evaluados, $37 \%$, que fue equivalente a 46 casos, presentó una visión de 20/16; $47 \%$, representado por 56 casos, obtuvo una visión 20/20; $8 \%$, correspondiente a 9 casos, evidenció una visión de 20/25; mientras que $3 \%$, equivalente a 3 casos, presentó una visión 20/30; y otro $3 \%$ una visión de 20/40. También hubo un $1 \%$ representado por 1 estudiante que obtuvo una visión de $20 / 50$ y otro $1 \%$ poseía 20/60; en cambio, ninguno de los estudiantes obtuvo una visión 20/100.

Tabla 1. Agudeza visual de ojo derecho en estudiantes según edad ( $\mathrm{n}=119)$

\begin{tabular}{|l|c|c|c|c|c|c|c|c|}
\hline \multicolumn{7}{|c|}{ Agudeza visual } \\
\hline Edad & $\mathbf{2 0 / 1 6}$ & $\mathbf{2 0 / 2 0}$ & $\mathbf{2 0 / 2 5}$ & $\mathbf{2 0 / 3 0}$ & $\mathbf{2 0 / 4 0}$ & $\mathbf{2 0 / 5 0}$ & $\mathbf{2 0 / 6 0}$ & $\mathbf{2 0 / 1 0 0}$ \\
\hline 8 años & 6 & 12 & 3 & 2 & 0 & 0 & 0 & 0 \\
\hline 9 años & 2 & 10 & 1 & 0 & 0 & 1 & 1 & 0 \\
\hline 10 años & 5 & 4 & 2 & 0 & 1 & 0 & 0 & 0 \\
\hline 11 años & 9 & 6 & 2 & 0 & 0 & 0 & 1 & 0 \\
\hline 12 años & 9 & 7 & 2 & 1 & 1 & 0 & 0 & 0 \\
\hline 13 años & 7 & 11 & 3 & 0 & 1 & 0 & 0 & 0 \\
\hline 14 años & 4 & 3 & 2 & 0 & 0 & 0 & 0 & 0 \\
\hline Total & $\mathbf{4 2}$ & $\mathbf{5 3}$ & $\mathbf{1 5}$ & $\mathbf{3}$ & $\mathbf{3}$ & $\mathbf{1}$ & $\mathbf{2}$ & $\mathbf{0}$ \\
\hline
\end{tabular}

Fuente: Bisonó et al., 2019. 
De acuerdo con los resultados mostrados en la tabla anterior, se llegó a la conclusión de que, según la edad de los estudiantes, aquellos que tenían ocho (8) ańos predominaron con la alteración de la agudeza visual del ojo derecho; de 23 estudiantes, tres presentaron una agudeza visual de $20 / 25$, y dos una agudeza visual de 20/30.

Tabla 2. Agudeza visual de ojo izquierdo en estudiantes según edad ( $\mathrm{n}=119)$

\begin{tabular}{|r|c|c|c|c|c|c|c|c|}
\hline \multicolumn{10}{|c|}{ Agudeza visual } \\
\hline Edad & $\mathbf{2 0 / 1 6}$ & $\mathbf{2 0 / 2 0}$ & $\mathbf{2 0 / 2 5}$ & $\mathbf{2 0 / 3 0}$ & $\mathbf{2 0 / 4 0}$ & $\mathbf{2 0 / 5 0}$ & $\mathbf{2 0 / 6 0}$ & $\mathbf{2 0 / 1 0 0}$ \\
\hline 8 años & 8 & 15 & 0 & 0 & 0 & 0 & 0 & 0 \\
\hline 9 años & 3 & 11 & 1 & 0 & 0 & 0 & 0 & 0 \\
\hline 10 años & 6 & 5 & 0 & 0 & 19 & 0 & 0 & 0 \\
\hline 11 años & 5 & 8 & 2 & 0 & 2 & 1 & 0 & 0 \\
\hline 12 años & 10 & 4 & 4 & 1 & 0 & 0 & 1 & 0 \\
\hline 13 años & 8 & 12 & 1 & 1 & 0 & 0 & 0 & 0 \\
\hline 14 años & 6 & 1 & 1 & 1 & 0 & 0 & 0 & 0 \\
\hline Total & $\mathbf{4 6}$ & $\mathbf{5 6}$ & $\mathbf{9}$ & $\mathbf{3}$ & $\mathbf{2 1}$ & $\mathbf{1}$ & $\mathbf{1}$ & $\mathbf{0}$ \\
\hline
\end{tabular}

Fuente: Bisonó et al., 2019.

De acuerdo con los resultados mostrados en la tabla anterior, se llegó a la conclusión de que, según la edad de los estudiantes, aquellos que tenían 12 años predominaron con la alteración de la agudeza visual del ojo izquierdo; cuatro estudiantes presentaron una agudeza visual de 20/25, uno presentó una agudeza visual de 20/30, y uno, una agudeza visual de 20/60.

Tabla 3. Presencia de signos y síntomas en los estudiantes $(\mathrm{n}=31)$

\begin{tabular}{|l|c|c|}
\hline \multicolumn{2}{|c|}{ Presencia de signos y síntomas en los estudiantes } \\
\hline \multicolumn{1}{|c|}{ Signos y síntomas } & Frecuencia & Porcentaje \\
\hline Cefalea & 24 & $77 \%$ \\
\hline Fotofobia & 11 & $35 \%$ \\
\hline Lagrimeo & 8 & $26 \%$ \\
\hline Prurito ocular & 11 & $35 \%$ \\
\hline Sequedad ocular & 1 & $3 \%$ \\
\hline Visión borrosa & 20 & $65 \%$ \\
\hline Visión doble & 2 & $6 \%$ \\
\hline Pterigium & 0 & $0 \%$ \\
\hline Ninguno & 3 & $10 \%$ \\
\hline
\end{tabular}

Fuente: Bisonó et al., 2019.

100 | Ciencia y Salud 2020; 4(3, septiembre-diciembre): Casos clínicos • 95-104 
De acuerdo con los resultados mostrados en la tabla anterior, se demuestran los signos y síntomas asociados a las alteraciones de la agudeza visual de los estudiantes de la escuela de El Carretón. Con base en los 31 estudiantes que presentaron alguna alteración de la agudeza visual, $77 \%$ padeció cefalea, siendo el mayor porcentaje, mientras que el $35 \%$ presentó fotofobia. Once (11) de los estudiantes, equivalente al $26 \%$, refirieron haber padecido de lagrimeo, y otros once (11) estudiantes, prurito ocular. Hubo un $3 \%$, equivalente a un (1) caso, que presentó sequedad ocular, y $65 \%$ de los estudiantes presentó visión borrosa; $6 \%$, equivalente a dos (2) casos, tuvieron visión doble, y ninguno de las pacientes presentó pterigium. Por último, $10 \%$ de los pacientes no presentó signos ni síntomas oculares. Cabe aclarar que varios de estos estudiantes presentaron más de un síntoma asociado a alteraciones de la agudeza visual.

Gráfica 3. Asistencia a consulta oftalmológica en los últimos 3 años ( $\mathrm{n}=119)$

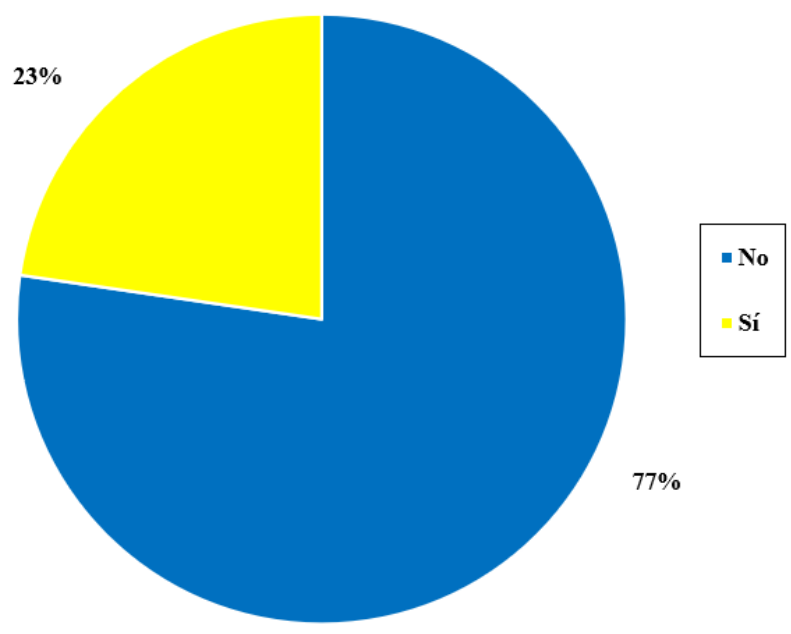

Fuente: Bisonó et al., 2019.

De acuerdo con los resultados presentados anteriormente, se observó que el $77 \%$ de la muestra no ha asistido durante los últimos 3 ańos a una consulta oftalmológica representando 92 estudiantes, en cambio el $23 \%$ de la muestra restante correspon- diente a 27 estudiantes, admitieron haber recibido una consulta oftalmológica en los últimos 3 años.

Tabla 4. Corrección de agudeza visual con agujero estenopeico en ojo derecho $(n=119)$

\begin{tabular}{|l|c|c|}
\hline \multicolumn{3}{|c|}{$\begin{array}{c}\text { Corrección de agudeza visual con agujero } \\
\text { estenopeico en ojo derecho }\end{array}$} \\
\hline $\begin{array}{c}\text { Corrigió } \\
\text { con agujero } \\
\text { estenopeico }\end{array}$ & Frecuencia & Porcentaje \\
\hline Sí & 8 & $7 \%$ \\
\hline No & 16 & $13 \%$ \\
\hline No aplica & 95 & $80 \%$ \\
\hline
\end{tabular}

Fuente: Bisonó et al., 2019.

De acuerdo con los resultados que se mostraron anteriormente, se observó que el $13 \%$ de la muestra, equivalente a 16 estudiantes, no mejoró su visión al colocarles el agujero estenopeico, en cambio, el $7 \%$ de la muestra restante, representado por 8 estudiantes, sí mejoró su visión al colocarle el mismo.

Tabla 5. Corrección de agudeza visual con agujero estenopeico en ojo izquierdo $(n=119)$

\begin{tabular}{|l|c|c|}
\hline \multicolumn{3}{|c|}{$\begin{array}{c}\text { Corrección de agudeza visual con agujero } \\
\text { estenopeico en ojo izquierdo }\end{array}$} \\
\hline $\begin{array}{c}\text { Corrigió } \\
\text { con agujero } \\
\text { estenopeico }\end{array}$ & Frecuencia & Porcentaje \\
\hline Sí & 6 & $5 \%$ \\
\hline No & 11 & $9 \%$ \\
\hline No aplica & 102 & $86 \%$ \\
\hline
\end{tabular}

Fuente: Bisonó et al., 2019. 
De acuerdo con los resultados que se mostraron anteriormente, se observó que el $9 \%$ de la muestra, equivalente a 11 estudiantes, no mejoró su visión al colocarles el agujero estenopeico, en cambio, el $5 \%$ de la muestra restante, conformado por seis (6) estudiantes, sí mejoró su visión al colocarle el mismo.

\section{Discusión y conclusiones}

Esta investigación mostró la prevalencia de alteración en la agudeza visual, discromatopsia y signos y síntomas asociados en una población estudiantil en la comunidad de El Carretón, Peravia, República Dominicana, revelando que la mayoría de los escolares no han asistido a alguna consulta oftalmológica previa, y que muchos de estos necesitan la misma. Según el presente estudio, el $77 \%$ de los estudiantes no han asistido a una consulta oftalmológica en los últimos tres años. De acuerdo a un artículo publicado por la American Academy of Ophthalmology en el 2015, los niños en edad escolar necesitan una evaluación de la agudeza visual cada año ${ }^{10}$. La realización de este tamizaje es difícil debido a que, en la Unidad de Atención Primaria de el Carretón, los médicos carecen de los materiales básicos para hacer un examen oftalmológico simple. Existen programas del Gobierno como "Ver para aprender" y "Programa visual de INABIE" que tiene como uno de sus objetivos realizar el tamizaje de la visión de los nińos de escuela pública en República Dominicana. Entre ambos proyectos, lograron a evaluar 258.000 estudiantes en el país. Desafortunadamente, estos programas se restringieron a regiones específicas, y el sector de el Carretón fue apartado del proyecto ${ }^{11,12}$. A la vez, de acuerdo con los datos arrojados por la investigación y la clasificación de la discapacidad de la OMS se exhibe que en la evaluación de la agudeza visual de ojo derecho de los estudiantes: $78 \%$ de los estudiantes tienen una visión normal y $28 \%$ una alteración de la agudeza visual. Dentro de este $28 \%, 19 \%$ tiene una discapacidad visual leve y $9 \%$ tiene una discapacidad moderada. En la evaluación del ojo izquierdo de los estudiantes, $84 \%$ de los estudiantes presenta una visión normal y $16 \%$ una alteración de la agudeza visual. Dentro de los 16 $\%, 14 \%$ tiene una alteración leve de la agudeza visual y $2 \%$ tiene una alteración moderada de la visión. Estos datos difieren de los encontrados en un estudio titulado Prevalencia de Defectos de Refracción en Escolares de Primero a Sexto Grado en el Municipio de Cane, La Paz, Honduras, en el año 2015 de Jorge Meléndez, donde se comprobó que aproximadamente $92 \%$ de los escolares evaluados tienen agudeza visual normal en ambos ojos, $6 \%$ poseen una agudeza visual levemente alterada y un $2 \%$ una alteración moderada de la agudeza visual ${ }^{13}$. Por otra parte, en las tablas 1 y 2 se puede apreciar que el rango de edad con más alteraciones de la vista del ojo derecho fueron los estudiantes de 12 años, mientras que los escolares de 8 años fueron los que más alteraciones de la vista presentaron en el ojo izquierdo. Estos valores difieren con los recolectados de una tesis titulada Valoración de la Disminución Agudeza Visual relacionada con alteraciones de la refracción en niños de 6-13 años en la Unidad Educativa Tres de Noviembre en el periodo 2016 realizado en Ecuador, donde el rango de edad más afectado en ambos ojos correspondió a los estudiantes de 7 años $^{14}$. A la vez, según las respuestas del cuestionario y plasmado en la tabla 3 , se demuestra que dentro de los signos y síntomas la cefalea fue el síntoma más frecuente, representando un $77 \%$ de los casos, seguido de visión borrosa con un 65\%. Lo anterior concuerda con los resultados de la investigación realizada en Honduras, donde se encontró que, del total de escolares con defectos de refracción, el síntoma principal fue la cefalea con un $32 \%$, seguido por prurito ocular con un $6 \%^{13}$. Por último, con la utilización del agujero estenopeico se obtuvo una mejoría de un 7 $\%$ en el ojo derecho en comparación con la del ojo izquierdo que fue un $5 \%$, predominó en ambos ojos la no mejoría de la visión con la utilización del instrumento en cuestión, puesto que se obtuvo que el $9 \%$ no mejoró en el ojo derecho y un $13 \%$ no mejoró en el izquierdo. Estos resultados discrepan de los de una investigación realizada en Ecuador, 
donde se observó que el $11.1 \%$ mejora su visión con el uso del agujero estenopeico en su ojo derecho mientras que solo un $2.0 \%$ no mejora con el uso del mismo y el $5.6 \%$ de los estudiantes mejora su déficit visual y solo el $1.5 \%$ del grupo de estudio no mejora con el empleo del agujero estenopeico ${ }^{14}$.

Se necesitan más investigaciones cualitativas para evaluar los determinantes clave con fin de aumentar la efectividad de los programas de atención ocular entre los escolares, así como para determinar el impacto que tiene la corrección de los errores de refracción causados por la discapacidad visual en el rendimiento escolar y la calidad de vida de los niños ${ }^{15}$.

\section{Bibliografía}

1. Pablo JV, Marcelo RS. Prevalencia de agudeza visual baja y trastornos oftalmológicos en niños de seis años de la ciudad de Santa Fe.. Arch. argent. Pediatr. 2008;106(4).

2. WHO. Word report on vision. [Internet]. Geneva, Switzerland. 2019. Disponible en: shorturl.at/lpwJ1

3. Vaughan y Asbury. Oftalmología general. (18a ed.). McGraw Hill; 2012

4. Ríos JD, Bettin L, Naranjo S, Suárez JA, De Vivero C. Pautas para el examen oftalmológico: enfoque para el estudiante de medicina y el médico general. Univ. Med. 2017;58. Doi: 10.11144/Javeriana.umed58-2.ofta

5. Araujo Chumacero MM, Solano Zapata FE, Vilela Estrada MA, Valladares Garrido MJ, Chumacero Aguilar M, Mejia CR. Factores socio-educativosasociadosalaagudezavisualbaja en escolares de Perú. Rev Pediatr Aten Primaria [Internet]. 2015;17(68):e261-e266. Disponible en: http://scielo.isciii.es/scielo.php?script=sci_ arttext\&pid=S1139-76322015000500005\&1ng=es. http://dx.doi.org/10.4321/S1139-7632 2015000500005.
6. Vázquez J. Exploración ocular en atención primaria. Semergen. 2002;28(5):265-74.

7. Tapia M. Caracterización de los problemas refractivos en niños de 6-12 años examinados en la clínica de optometría del CICS ST, en el periodo enero-diciembre 2009. México: Instituto Politécnico Naacional; 2010. Disponible en: https://tesis.ipn.mx/ handle/123456789/9510

8. CapetilloBiartO,Triana Casado I, MartínezLegón Z, Roche Caso S, Broche HernándezA. Frecuencia de la ambliopía en escolares. Rev Cubana Pediatr [Internet]. 2011;83(4):372-81. Disponible en: http://scielo.sld.cu/scielo.php?script=sci_arttext\&pid=S0034-75312011000400005\&lng=es.

9. American Academy of Ophthalmology. Pediatric Eye Evaluations PPP [Internet]. Elsevier Inc. 2017 Disponible en: https://www.aao.org/ preferred-practice-pattern/pediatric-eye-evaluations-ppp-2017

10. Frequency of Ocular Examinations - 2015 [Internet]. American Academy of Ophthalmology. 2019. Disponible en: https://www. aao.org/clinical-statement/frequency-of-ocular-examinations

11. Más de 200 mil estudiantes se benefician de programa "Ver Bien para Aprender" - Periódico El Caribe - Mereces verdaderas respuestas [Internet]. 2019. Disponible en: https://www.elcaribe.com. do/2017/02/03/mas-200-mil-estudiantes-benefician-programa-ver-bien-para-aprender

12. Programa de salud visual de Inabie impacta más de 58 mil estudiantes de Duarte [Internet]. Hoy Digital. 2019. Disponible en: https://hoy.com. do/programa-de-salud-visual-de-inabie-impacta-mas-de-58-mil-estudiantes-de-duarte 
13. Melendez J, Aviles S, Erazo Recinos A, Galo Canaca S. Prevalencia de Defectos de Refracción en Escolares de Primero a Sexto Grado en el Municipio de Cane La Paz Honduras en el año 2015 [Internet]. Archivosdemedicina.com. 2019. Disponible en: http://www.archivosdemedicina.com/medicina-de-familia/prevalencia-de-defectos-de-refraccioacuten-en-escolares-de-primero-a-sexto-grado-en-el-municipio-de-cane-la-paz-honduras-en-el-ant.pdf

14. Pazmiño Martínez M, Piña Bermeo S. Valoración de la Disminución Agudeza Visual relacionada con alteraciones de la refracción en niños de 6-13 años en la Unidad Educativa Tres de Noviembre en el periodo 2016 [Internet]. Dspace.ucuenca. edu.ec. 2015. Disponible en: http://dspace. ucuenca.edu.ec/bitstream/123456789/25939/1/ PROYECTO\%20DE\%20INVESTIGACI\%C3\%93N.pdf
15. Schneider J, Leeder SR, Gopinath B, Wang JJ, MitchellP. Frequency, course, and impact of correctable visual impairment (uncorrected refractive error). Surv Ophthalmol. 2010;55(6):539-60. Disponible en: https://www.surveyophthalmol. com/article/S0039-6257(10)00042-1/fulltext 